\title{
Thrombotic complications of vaccination against SARS-CoV-2: what pharmacovigilance reports tell us - and what they don't
}

\author{
Stavros V. Konstantinides $\mathbb{1}^{1,2}$ \\ ${ }^{1}$ Center for Thrombosis and Hemostasis, University Medical Center of the Johannes Gutenberg University, Mainz, Germany. ${ }^{2}$ Dept of \\ Cardiology, Democritus University of Thrace, Alexandroupolis, Greece.
}

Corresponding author: Stavros V. Konstantinides (stavros.konstantinides@unimedizin-mainz.de)

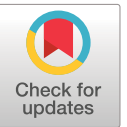

Copyright $\odot$ The authors 2021

This version is distributed under the terms of the Creative Commons Attribution Non-Commercial Licence 4.0. For commercial reproduction rights and permissions contact permissions@ersnet.org

Received: 17 April 2021 Accepted: 19 April 2021

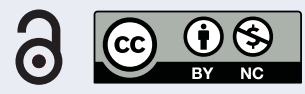

Shareable abstract (@ERSpublications)

Thrombotic complications of COVID-19 vaccination are very rare, but awareness is needed https://bit.ly/3n4vaXe

Cite this article as: Konstantinides SV. Thrombotic complications of vaccination against SARS-CoV-2: what pharmacovigilance reports tell us - and what they don't. Eur Respir J 2021; 58: 2101111 [DOI: 10.1183/13993003.01111-2021].

In the present issue of the European Respiratory Journal, SMADJA et al. [1] present an analysis of global pharmacovigilance reports of thrombotic events following severe acute respiratory distress syndrome coronavirus 2 (SARS-CoV-2) vaccination. More specifically, the authors analysed the data entered into the World Health Organization (WHO) Global Database for Individual Case Safety Reports (VigiBase) between 13 December, 2020 and 16 March, 2021, covering, at that time, a population of almost 362 million vaccinated individuals across the world. The study focuses on three of the coronavirus disease 2019 (COVID-19) vaccines available to date, namely Pfizer-BioNtech (BNT162b2), Moderna (mRNA-1273) and OxfordAstraZeneca (ChAdOx1 nCov-19). The study by SMADjA et al. [1] confirms the rarity of possible thrombotic complications in association with COVID-19 vaccination, reporting only 0.21 (95\% CI 0.190.22) cases of thrombotic events per million person vaccinated-days. However, there were also some unexpected observations which deserve closer attention and cautious interpretation.

From the beginning of the COVID-19 pandemic, this disease has been associated with frequent thrombotic complications, due to the direct effects of the virus on the endothelium and to the "cytokine storm" following the host's immune response [2, 3]. In a recent meta-analysis of 102 studies involving 64503 patients, the frequency of COVID-19-related venous thromboembolism was as high as $14.7 \%$ (95\% CI 12.1-17.6\%) and that of COVID-19-related arterial thrombotic events 3.9\% (95\% CI 2.0-3.0\%) [4]. The cumulative rate of thromboembolic events is particularly high among critically ill patients necessitating admission to an intensive care unit $[5,6]$. Very recently, a small study reported a $14.2 \%$ (95\% CI 7.5 $20.8 \%$ ) prevalence of pulmonary embolism at the time of hospital admission for COVID-19, further underscoring the thromboembolic risk in this setting [7]. Consequently, since the COVID-19 vaccines mentioned above have been shown to effectively prevent symptomatic infection including hospital admissions and severe disease [8-10], the risk of COVID-19-related thrombosis should be expected to (almost) disappear in vaccinated individuals.

Just a few weeks ago, however, COVID-19-related thrombosis returned, through the back door. Beginning in late February 2021, a small but increasing number of thrombotic events, often at unusual sites such as the sinus or splanchnic veins, and often in association with thrombocytopenia, were reported in relation to the adenoviral vector-based ChAdOx1 nCov-19 vaccine, immediately sparkling intense scientific (and political) debates. Use of the vaccine was suspended in several European countries, only to partly resume after a statement by the European Medicines Agency, which confirmed that "the benefits of the vaccine in combating the COVID-19 outbreak continue to outweigh the risk of side effects.” The agency has thus far reviewed 62 cases of cerebral venous sinus thrombosis and 24 cases of splanchnic vein thrombosis, with a total of 18 fatal thrombotic events, reported in the EudraVigilance drug safety database among 25 million vaccinated individuals as of 22 March, 2021. Meanwhile, the number of reported cases has risen to 169 
possible cases of cerebral venous sinus thrombosis and 53 possible cases of splanchnic vein thrombosis among 34 million recipients of the ChAdOx1 nCoV-19 vaccine.

Expert groups moved quickly, and the pathogenesis of these obscure thrombotic events could be elucidated, at least in part. Very recently, a publication from Germany and Austria reported that laboratory analysis of blood samples from 11 patients with post-vaccination (with ChAdOx1 nCov-19) thrombotic events yielded evidence of immune thrombotic thrombocytopenia mediated by platelet-activating antibodies against platelet factor 4 [11]. The pathomechanisms are reminiscent of autoimmune heparin-induced thrombocytopenia, although none of the patients included in the report had received heparin [11]. An analysis of the samples from five patients in Norway, who presented with venous thrombosis and thrombocytopenia 7 to 10 days after receiving the first dose of the same vaccine, yielded similar findings [12], as did a report on 23 cases from the UK [13]. Overall, approximately $40 \%$ of the studied patients died, either from ischaemic brain injury or from superimposed haemorrhage. This new-born, potentially life-threatening clinical entity already has a proposed name, "vaccine-induced immune thrombotic thrombocytopenia (VITT)".

The analysis by SMADJA et al. [1] provides new, potentially relevant insights into the field of vaccine-related thrombosis. In a global pharmacovigilance database including a 15 times larger vaccinated population than that reviewed by the EMA and focusing on three vaccines, rather than one in particular, rare reports of thrombotic complications now also appear in relation to the mRNA-based Pfizer-BioNtech and Moderna vaccines. If these (and any future) reports are confirmed, and VITT supported by thorough laboratory testing, as were the few cases described above, they may trigger investigation of further mediators of this aberrant immune response, searching beyond the adenoviral sequence or other components of the particular ChAdOx1 nCoV-19 (AstraZeneca) and, possibly, the Ad26.COV2.S (Johnson \& Johnson/Janssen) adenoviral vector vaccine.

As emphasised in the beginning, both the frequency and the absolute number of events, including thrombosis-related deaths, remain extremely low if viewed against the global impact of COVID-19 itself with 140 million cases and 3.0 million deaths thus far [14], and when put into the perspective of more than 751 million individuals vaccinated worldwide [15] as of 16 April, 2021. Moreover, and importantly, data such as those analysed by SMADJA et al. [1] should be interpreted with extreme caution due to numerous important limitations, which are highlighted by the authors. Pharmacovigilance reports contain administrative, non-adjudicated data which can help to generate hypotheses, but they cannot, and should not, support speculation on causal relations. In addition, the present analysis does not permit any direct comparisons between the three studied vaccines with regard to the risk for VITT, since only the numerator, i.e. the number of thrombosis reports, but not the denominator for each vaccine, i.e. the total number of the recipients of the particular vaccine for which thrombotic events were reported, is given. This must always be kept in mind when reading table 1 of the report by SMADJA et al. [1]. Finally, the likelihood of both underreporting and overreporting of adverse events to the WHO is not to be underestimated. Thus, in view of all the things that the present publication does not tell us, it must not be interpreted as challenging the safety of any COVID-19 vaccine and should not be used as an argument to halt or slow the life-saving global vaccination programme. On the other hand, the results of SMADJA et al. [1] do highlight the importance of continuing pharmacovigilance after the approval of any vaccine (and any drug), in everyday clinical practice. Analysing pharmacovigilance reports helps to increase awareness of authorities and physicians to specific alarming clinical symptoms and signs, so that these can be detected and managed early, however rare they may be. In the future, improving insights into the pathogenesis of VITT may help us to better select candidates for specific vaccines, minimise uncertainty in the medical community and the lay public, and help us to further accelerate the global vaccination programme, and do so safely, with the aim of liberating the population from the COVID-19 pandemic.

Conflict of interest: S.V. Konstantinides has received fees for consultancy and lecture honoraria from Bayer HealthCare, Boehringer Ingelheim, Actelion, Servier and BMS Pfizer; reimbursement for travel, accommodation and meeting expenses from Bayer HealthCare; and institutional grants from Boehringer Ingelheim, Bayer HealthCare, Daiichi Sankyo and Actelion.

Support statement: The work of S.V. Konstantinides was supported by the German Federal Ministry of Education and Research (BMBF 01EO1003 and 01EO1503). The author is responsible for the contents of this publication.

\section{References}

1 Smadja D, Yue Q-Y, Chocron R, et al. Vaccination against COVID-19: insight from arterial and venous thrombosis occurrence using data from VigiBase. Eur Respir J 2021; 58: 2100956. 
2 Ackermann M, Verleden SE, Kuehnel M, et al. Pulmonary vascular endothelialitis, thrombosis, and angiogenesis in Covid-19. N Engl J Med 2020; 383: 120-128.

3 Connors JM, Levy JH. COVID-19 and its implications for thrombosis and anticoagulation. Blood 2020; 135: 2033-2040.

4 Tan BK, Mainbourg S, Friggeri A, et al. Arterial and venous thromboembolism in COVID-19: a study-level meta-analysis. Thorax 2021; in press [https://doi.org/10.1136/thoraxjnl-2020-215383].

5 Lodigiani C, lapichino G, Carenzo L, et al. Venous and arterial thromboembolic complications in COVID-19 patients admitted to an academic hospital in Milan, Italy. Thromb Res 2020; 191: 9-14.

6 Klok FA, Kruip M, van der Meer NJM, et al. Confirmation of the high cumulative incidence of thrombotic complications in critically ill ICU patients with COVID-19: an updated analysis. Thromb Res 2020; 191: 148-150.

7 Jevnikar M, Sanchez O, Chocron R, et al. Prevalence of pulmonary embolism in patients with COVID 19 at the time of hospital admission. Eur Respir J 2021; 58: 2100116.

8 Polack FP, Thomas SJ, Kitchin N, et al. Safety and efficacy of the BNT162b2 mRNA Covid-19 vaccine. N Engl J Med 2020; 383: 2603-2615.

9 Voysey M, Costa Clemens SA, Madhi SA, et al. Single-dose administration and the influence of the timing of the booster dose on immunogenicity and efficacy of ChAdOx1 nCoV-19 (AZD1222) vaccine: a pooled analysis of four randomised trials. Lancet 2021; 397: 881-891.

10 Baden LR, El Sahly HM, Essink B, et al. Efficacy and safety of the mRNA-1273 SARS-CoV-2 vaccine. N Engl J Med 2021; 384: 403-416.

11 Greinacher A, Thiele T, Warkentin TE, et al. Thrombotic thrombocytopenia after ChAdOx1 nCov-19 vaccination. N Engl J Med 2021; 384: 2092-2101.

12 Schultz $\mathrm{NH}$, Sorvoll IH, Michelsen AE, et al. Thrombosis and thrombocytopenia after ChAdOx1 nCoV-19 vaccination. N Engl J Med 2021; 384: 2124-2130.

13 Scully M, Singh D, Lown R, et al. Pathologic antibodies to platelet factor 4 after ChAdOx1 nCoV-19 vaccination. N Engl J Med 2021; 384: 2202-2211.

14 Johns Hopkins University and Medicine. Coronavirus Resource Center. https://coronavirus.jhu.edu/ Date last accessed: 17 Apr 2021.

15 World Health Organization. WHO Coronavirus (COVID-19) Dashboard. https://covid19.who.int/ Date last accessed: 17 Apr 2021. 